INPLASY

PROTOCOL

To cite: Meza-Mauricio et al. How Efficacious is the Combination of Substitute Bone Graft with Autogenous Bone Graft in Comparison with Substitute Bone Graft Alone in the horizontal bone gain? A Systematic Review and Metaanalysis. Inplasy protocol 2021800109. doi: 10.37766/inplasy2021.8.0109

Received: 28 August 2021

Published: 28 August 2021

Corresponding author: Jonathan Meza-Mauricio

jomemau60@gmail.com

Author Affiliation:

Guarulhos university

Support: Guarulhos university.

Review Stage at time of this submission: Data analysis.

Conflicts of interest:

None declared.

\section{How Efficacious is the Combination of Substitute Bone Graft with Autogenous Bone Graft in Comparison with Substitute Bone Graft Alone in the horizontal bone gain? A Systematic Review and Meta-analysis}

Meza-Mauricio, J1; Furquim, CP2; Delfino dos Reis, L3; Maximiano, $\mathrm{MMH}^{4}$; Mendoza-Azpur G5; Muniz, FWMG6; Rasperini, $\mathrm{G}^{7}$; Faveri $\mathrm{M}^{8}$.

Review question / Objective: In patients who were submitted to horizontal guided bone regeneration, how efficacious is the combination of substitute bone graft with autogenous bone graft in comparison to substitute bone graft alone, in terms of bone gain?

Condition being studied: To evaluated how efficacious is the combination of substitute bone graft with autogenous bone graft in comparison to substitute bone graft alone. Information sources: The grey literature in the System for Information on Grey Literature in Europe (http:// www.opengrey.eu) and The New York Academy of Medicine Grey Literature Report (http://www.greylit.org) were electronically screened, as recommended by the high standards for systematic reviews (AMSTAR guideline). Furthermore, hand searches of relevant primary sources related to the topic were performed in Clinical Implants Dentistry and Related Research, Journal of Clinical Periodontology, Clinical Oral Implants Research and Clinical Oral Investigations. Finally, the list of references of studies included were also hand searched to capture any potential additional records, as suggested by Greenhalgh andPeacock.

INPLASY registration number: This protocol was registered with the International Platform of Registered Systematic Review and Meta-Analysis Protocols (INPLASY) on 28 August 2021 and was last updated on 28 August 2021 (registration number INPLASY202180109).

\section{INTRODUCTION}

Review question / Objective: In patients who were submitted to horizontal guided bone regeneration, how efficacious is the combination of substitute bone graft with autogenous bone graft in comparison to 
substitute bone graft alone, in terms of bone gain?

Condition being studied: To evaluated how efficacious is the combination of substitute bone graft with autogenous bone graft in comparison to substitute bone graft alone.

\section{METHODS}

Search strategy: The MEDLINE (PubMed), Embase, Scopus, and Web of Science databases were searched up to March 2021 by two independent reviewers (J.M.M. and C.P.F.). The search was performed without restrictions on dates or language. The search strategy was applied as follows: PubMed: ( "Horizontal ridge deficiencies"[All Fields] OR "horizontal alveolar ridge augmentation"[All Fields] OR "lateral ridge augmentation"[All Fields] OR "Lateral bone augmentation"[All Fields] OR "Horizontal bone augmentation"[All Fields] OR "horizontal Bone regeneration"[All Fields] OR "Horizontal ridge augmentation"[All Fields]) AND ("Bone graft"[All Fields] OR "Autologous bone"[All Fields] OR "Autogenous bone"[All Fields] OR "Bone substitute"[All Fields] OR "Allograft bone"[All Fields] OR "Allogenic bone"[All Fields] OR "Xenogeneic bone"[All Fields] OR "Alloplastic"[All Fields]).

Participant or population: Patients with horizontal atrophic alveolar ridge in need of horizontal guided bone regeneration prior to dental implant installation.

Intervention: Bone augmentation using guide bone regeneration. This procedure needed to have been performed with particulate materials, such as autologous bone chips, and/or osteoconductive materials, such as allografts, xenografts, or alloplastic bone substitute materials.

Comparator: Substitute bone graft + autogenous bone graft vs substitute bone graft alone.

Study designs to be included: Randomized clinical trial (RCTs).
Eligibility criteria: The inclusion criteria were based on the PICOS strategy.

Information sources: The grey literature in the System for Information on Grey Literature in Europe (http:// www.opengrey.eu) and The New York Academy of Medicine Grey Literature Report (http://www.greylit.org) were electronically screened, as recommended by the high standards for systematic reviews (AMSTAR guideline). Furthermore, hand searches of relevant primary sources related to the topic were performed in Clinical Implants Dentistry and Related Research, Journal of Clinical Periodontology, Clinical Oral Implants Research and Clinical Oral Investigations. Finally, the list of references of studies included were also hand searched to capture any potential additional records, as suggested by Greenhalgh andPeacock.

\section{Main outcome(s): Horizontal bone gain.}

Additional outcome(s): Graft resorption, and histological findings and patientreported outcome measurements (PROMs) (pain, discomfort, satisfaction, etc.)

Quality assessment / Risk of bias analysis: Two reviewers (C.P.F and J.M.M) assessed the risk of bias in the studies selected, using the Cochrane risk-of-bias tool, RoB 2 (version 2, available at: https:// www.riskofbias.info/welcome/rob-2-0-tool/ current-version-of-rob-2). The authors of this systematic review decided to assess the result related to "assignment to intervention (the intention-to-treat effect)" and five domains were examined: (i) bias arising from the process of randomization and allocation concealment, (ii) bias due to deviations from intended interventions that involved masking of participants and our team of researchers, (iii) bias due to missing outcome data, (iv) bias in measurement of the outcome, and (v) bias in selection of the result reported. Based on the answers to signaling questions and algorithms of this tool, each domain was judged as presenting "low risk of bias", "some concerns relating to the risk of bias," or "high risk of bias". Studies were 
categorized as being at low risk of bias (all domains were at low risk of bias), high risk of bias (one or more domains were at high risk of bias), some concerns (if one or more domains had some concerns). Disagreements were resolved by discussion, consulting a third researcher (G.R.).

Strategy of data synthesis: The software Rev-Man (version 5.3 for Windows, The Cochrane Collaboration, Copenhagen) was used to perform both meta-analyses. Statistical heterogeneity between both groups was assessed using Cochran's Qtest, with a threshold P-value of 0.1 , and the inconsistency 12 test, in which values $>\mathbf{5 0} \%$ were considered indicative of high heterogeneity.

Subgroup analysis: As the methodological characteristics differed among the studies included (some studies have used acellular dermal matrix and other used collagen membrane), both analyses were performed using a random effect model.

Sensitivity analysis: Sensitivity analysis was no performed.

Country(ies) involved: Brazil, Italy and Peru.

Keywords: bone graft; bone regeneration; allograft.

Contributions of each author:

Author 1 - Jonathan Meza-Mauricio Performed the search in data base, selected of the included papers, evaluated the risk of bias in the included studies and drafted the manuscript.

Email: jomemau60@gmail.com

Author 2 - Camila Pinheiro Furquim Performed the search in data base, selected of the included papers, evaluated the risk of bias in the included studies and drafted the manuscript.

Email: camilapfurquim@yahoo.com.br

Author 3 - Leonardo Delfino dos Reis Performed the data extraction and drafted the manuscript.

Email: leodreis@gmail.com
Author 4 - Marlon Marx Hilariano Maximiano - Performed the data extraction and drafted the manuscript.

Email: marlonhmaximiano@gmail.com Author 5 - Gerardo Mendoza-Azpur Performed the selected of included studies and drafted the manuscript.

Email: drgerardoodonto@yahoo.com

Author 6 - Francisco Wilker Mustafa Gomes Muniz - Performed the metaanalysis and drafted the manuscript.

Email: wilkermustafa@gmail.com

Author 7 - Giulio Rasperini - Third advisor in the evaluation of risk of bias of included studies, writing review and editing of the current manuscript.

Email: giulio.rasperini@unimi.it

Author 8 - Marcelo Faveri - Third advisor in the evaluation of risk of bias of included studies, writing review and editing of the current manuscript.

Email: mdfaveri@uol.com.br 